\title{
The Weird Wiggle of Polymers
}

\author{
According to the results of new neutron scattering experiments, polymer \\ molecules in plastics move in ways that aren't captured by commonly \\ used models.
}

By Sophia Chen

M

elt a plastic, and its constituent molecules, known as polymers, wiggle around. Experts typically describe polymer motion using the so-called tube model, which imagines plastics as a tangle of polymer strands-think a bowlful of worms. The model assumes that each strand moves independently within a virtual tube. Now, Michaela Zamponi of the Jülich Research Centre in Germany and colleagues have observed strand motion in a polymer melt that contradicts the idea of independent motion [1]. The results build on other studies that challenge the tube model (see Viewpoint: Tube Model Under Tension) and could help researchers refine theoretical concepts of polymer behaviors.

The team used neutron scattering spectroscopy to study a polymer melt containing a few short polyethylene chains in a sea of long strands of the same material. Because the long polyethylene strands contain a heavier isotope of hydrogen than the short chains, the neutrons scatter differently off the two types of strands, allowing the researchers to follow the motion of the short strands.

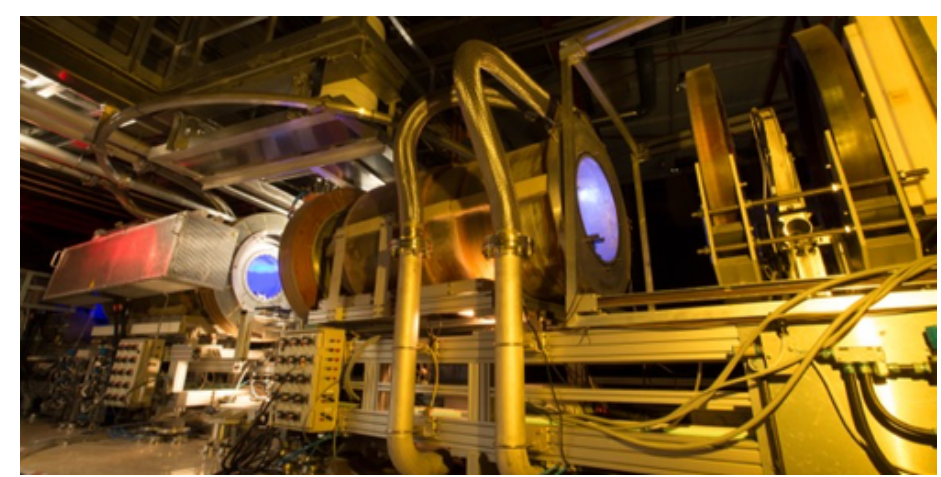

Credit: B. Cubitt/Institut Laue-Langevin

Monitoring the center of mass motion of the short strands, they observed two behavior regimes. For short translational distances, the motion of the short strands slowed as they grew apart. For longer distances, when the center of mass of the strands reached a size on the order of the diameter of the virtual tube, the speed at which the short strands moved stopped slowing down and instead matched that of diffusion.

They also found that the motions of short strands were tied to those of neighboring strands at short distances, differing from a standard assumption of the tube model. This cooperative motion may come from interactions between the segments, beyond simple local friction.

Sophia Chen is a freelance science writer based in Columbus, Ohio.

\section{REFERENCES}

1. M. Zamponi et al., "Cooperative chain dynamics of tracer chains in highly entangled polyethylene melts," Phys. Rev. Lett. 126, 187801 (2021). 